Theoretical Mathematics \& Applications, Vol. 10, No. 4, 2020, 1-5

ISSN: 1792-9687(print), 1792-9709(online)

https://doi.org/10.47260/tma/1041

Scientific Press International Limited

\title{
A Purely Mathematical Proof of Riemann Hypothesis
}

\author{
Robert Deloin ${ }^{1}$
}

\begin{abstract}
Riemann hypothesis $(\mathrm{RH})$ is the conjecture that the real part of every non-trivial zero of the Riemann zeta function is $1 / 2$.

The main contribution of this paper is to achieve the proof of Riemann hypothesis. The key idea to do it is to choose a counter-hypothesis to RH and show that it leads to a (double) contradiction.
\end{abstract}

MSC 2010 classification numbers: Primary 11A41, 11M06, 11M26.

Keywords: Riemann Hypothesis, Prime, Conjecture.

${ }^{1}$ Independent researcher. 13320 Bouc Bel Air, France. 


\section{Introduction}

In his book [1] of 1748 Euler proved what is now named the Euler product formula. This product is the result of the infinite sum

$$
\sum_{\mathrm{n}=1}^{\infty} 1 / n^{s}=\Pi_{p \text { in }\{P\}}\left(1-1 / p^{s}\right)^{-1} \text { for any integer variable } s>1
$$

where $\{\mathrm{P}\}$ is the infinite set of primes.

In his article [2] of 1859 Riemann extended Euler's result to the complex variable s of the zeta function

$$
\zeta(s)=\Pi_{p} \text { in }\{P\}\left(1-1 / p^{s}\right)^{-1} \text { for any complex variable } s \neq 1
$$

It is known that the real zeros of the function are the infinite set

$$
\left\{s_{1}\right\}=\{-2 m\} \quad \text { for all integers } m>0 .
$$

Riemann hypothesis $(\mathrm{RH})$ can be seen as stating that

Probably, the infinite set $\left\{s_{2}\right\}$ of non-trivial zeros of $\zeta(s)$ can be written

$$
\left\{s_{2}\right\}=\left\{1 / 2+i t_{n}\right\} \text { where } t_{n} \text { is real. }
$$

This conjecture is the first point of the eighth unresolved problem (among 23) that Hilbert listed in 1900 [3] as well as the second unresolved problem listed in 2000 by the Clay Mathematics Institute [4]. As of 2020, this conjecture was still open. It has been very well described by Bombieri [5].

\section{Proof of Riemann Hypothesis}

Various approaches were listed by Conrey [6] who says in his conclusion:

"A major difficulty in trying to construct a proof of RH through analysis is that the zeros of L-functions behave so much differently from zeros of many of the special functions we are used to seeing in mathematics and mathematical physics."

That is why in the next lines, the many attempts of proof based on analysis of Lfunctions are consciously ignored and an approach is chosen, based on the basics of complex numbers. This proof is largely inspired by Deloin [7].

Proof. By definition, a complex number $s$ is written

$$
s=x+i y \text { where } x \text { and } y \text { are real and } i=\sqrt{ }-1
$$

By changing the conventional coordinate system $(x, y)$ of the complex plane into the new one $\left(x^{\prime}=a-x, y^{\prime}=y\right)$ where $a$ is any finite real number, the complex numbers can be written either

$$
s^{\prime}=x^{\prime}+i y^{\prime} \text { in the new system }
$$


or, using the change of coordinates

$$
s^{\prime}=(a-x)+i y
$$

Hypothesis. Now, we suppose that the infinitely many non-trivial zeros $s_{2}$ of $\zeta(s)$ (with $y_{2}=t_{n}$ ) can lie at any $x_{2}$ in the complex plane with the two exceptions that they cannot lie on the real axis $x$ or $x^{\prime}$ (reserved for trivial zeros $s_{1}$ ), which gives

$$
y \neq 0 \text { and } y^{\prime} \neq 0
$$

nor on a unique vertical line $x=a$ (where $a$ is any real number) that becomes the new imaginary axis $y^{\prime}$ by the change of coordinates. This gives

$$
x \neq a \text { and } x^{\prime} \neq 0
$$

Then, each non-trivial zero $s_{2}$ of $\zeta(s)$ could be written

$$
s_{2}^{\prime}=x_{2}^{\prime}+i y_{2}^{\prime} \quad \text { with } x_{2}^{\prime} \neq 0 \text { and } y_{2}^{\prime} \neq 0
$$

or, using the change of coordinates

$$
s_{2}^{\prime}=\left(a-x_{2}\right)+i y_{2} \quad \text { with } x_{2} \neq a \text { and } y 2 \neq 0
$$

Now, using the fact that $-x_{2}=i^{2} x_{2}$, they could also be written

$$
s_{2}^{\prime}=\left(a+i^{2} x_{2}\right)+i y_{2}=a+i\left(y_{2}+i x_{2}\right) \quad \text { with } x_{2} \neq a \text { and } y_{2} \neq 0
$$

or

$$
s_{2}^{\prime}=a+i t_{2} \quad \text { with } t_{2}=y_{2}+i x_{2}, x_{2} \neq a \text { and } y_{2} \neq 0
$$

and we get the result, as $t_{2}$ has to be real, that $x_{2}$ has to be zero

$$
x_{2}=0
$$

But this result $x_{2}=0$ is wrong for two reasons.

1- It means that the infinite set of non-trivial zeros has to be located on a unique vertical line $x_{2}=0$ which is a contradiction to our hypothesis that excludes vertical lines by $x_{2} \neq a$ ( $a$ being any real number),

2- This result $x_{2}=0$ has been proven wrong $10^{13}$ times by Gourdon and Demichel [8] for the first $10^{13}$ non-trivial zeros $s_{2}$ for which $x_{2}=1 / 2$.

These two reasons mean that our hypothesis is wrong and respectively imply that 


$$
x_{2}=a \quad \text { and } \quad x_{2}=1 / 2
$$

and thus that

$$
x_{2}=a=1 / 2
$$

Finally, putting back this result in (7) with $y_{2}=t_{n}$ we get

$$
s_{2}^{\prime}=\left(a-x_{2}\right)+i y_{2}=i t_{n}
$$

or, back in the conventional complex plane

$$
s_{2}=x_{2}+i y_{2}=1 / 2+i t_{n}
$$

which proves Riemann hypothesis.

\section{Conclusion}

It has to be noticed and highlighted that this proof does not use, as guessed by Conrey, any of the classical and internal analysis of $\zeta(s)$ with integrals, L-functions, Fourier series, probabilities, etc. It uses only complex numbers, in response to the complex variable $s$ used by Riemann.

ACKNOWLEDGEMENTS. This work is dedicated to my family. 


\section{References}

[1] Euler, L. (1748). Introductio in analysin infinitorum. Euler Archive - All Works, 101 and 102. https://scholarlycommons.pacific.edu/euler-works/101/ and https://scholarlycommons.pacific.edu/euler-works/102/

[2] Riemann, B. (1859). On the Number of Prime Numbers less than a Given Quantity. Translation by Wilkins D.R. (1998). http://www.claymath.org/sites/default/files/ezeta.pdf

[3] Hilbert, D. (1900). Mathematische Probleme. Göttinger Nachrichten, pp. 253-297.

[4] Clay Mathematics Institute, (2000). Oxford UK. http://www.claymath.org/millennium-problems/riemann-hypothesis

[5] Bombieri, E., (2000). The Riemann Hypothesis - official problem description. Clay Mathematics Institute, Oxford UK. http://www.claymath.org/millennium-problems/riemann-hypothesis

[6] Conrey, J. B., (2003). The Riemann Hypothesis. Notices of the American Mathematical Society, pp. 341-353. http://www.ams.org/notices/200303/fea-conrey-web.pdf

[7] Deloin, R., (2018). Proof of Riemann Hypothesis. Asian Research Journal of Mathematics, 9(1), pp. 1-8. DOI: 10.9734/ARJOM/2018/40341. https://www.journalarjom.com/index.php/ARJOM/article/view/24575/45944

[8] Gourdon, X. and Demichel, P. (2004). Computation of zeros of the Zeta function.

http://numbers.computation.free.fr/Constants/Miscellaneous/zetazeroscomput e.html and more specically the "paper" linked to this web page. 